\title{
Vibrio campbellii hmgA-mediated pyomelanization impairs quorum sensing, virulence, and cellular fitness
}

\author{
Zheng Wang ${ }^{1}$, Baochuan Lin ${ }^{1}$, Anahita Mostaghim ${ }^{1,2}$, Robert A. Rubin ${ }^{3}$, Evan R. Glaser ${ }^{4}$, \\ Pimonsri Mittraparp-arthorn ${ }^{5}$, Janelle R. Thompson ${ }^{6}$, Varaporn VuddhakuI ${ }^{5}$ and Gary J. Vora ${ }^{1}$ \\ ${ }^{1}$ Center for Bio/Molecular Science \& Engineering, Naval Research Laboratory, Washington, DC, USA \\ 2 School of Systems Biology, College of Science, George Mason University, Fairfax, VA, USA \\ ${ }^{3}$ Mathematics Department, Whittier College, Whittier, CA, USA \\ ${ }^{4}$ Division of Electronics Science and Technology, Naval Research Laboratory, Washington, DC, USA \\ ${ }^{5}$ Department of Microbiology, Faculty of Science, Prince of Songkla University, Hat Yai, Thailand \\ ${ }^{6}$ Department of Civil and Environmental Engineering, Massachusetts Institute of Technology, Cambridge, MA, USA
}

\section{Edited by:}

Daniela Ceccarelli, University of

Maryland, USA

Reviewed by:

Zongze Shao, State Oceanic

Administration, China

Xiu-Lan Chen, Shandong university, China

*Correspondence:

Zheng Wang, Center for Bio/Molecular Science \&

Engineering, Naval Research

Laboratory, Code 6900, 4555

Overlook Avenue, SW, Washington,

DC 20375, USA

e-mail: zheng.wang@nrl.navy.mil
Melanization due to the inactivation of the homogentisate-1,2-dioxygenase gene (hmgA) has been demonstrated to increase stress resistance, persistence, and virulence in some bacterial species but such pigmented mutants have not been observed in pathogenic members of the Vibrio Harveyi clade. In this study, we used Vibrio campbellii ATCC BAA-1116 as model organism to understand how melanization affected cellular phenotype, metabolism, and virulence. An in-frame deletion of the hmgA gene resulted in the overproduction of a pigment in cell culture supernatants and cellular membranes that was identified as pyomelanin. Unlike previous demonstrations in Vibrio cholerae, Burkholderia cepacia, and Pseudomonas aeruginosa, the pigmented $V$. campbellii mutant did not show increased UV resistance and was found to be $\sim 2.7$ times less virulent than the wild type strain in Penaeus monodon shrimp virulence assays. However, the extracted pyomelanin pigment did confer a higher resistance to oxidative stress when incubated with wild type cells. Microarray-based transcriptomic analyses revealed that the hmgA gene deletion and subsequent pyomelanin production negatively effected the expression of 129 genes primarily involved in energy production, amino acid, and lipid metabolism, and protein translation and turnover. This transcriptional response was mediated in part by an impairment of the quorum sensing regulon as transcripts of the quorum sensing high cell density master regulator LuxR and other operonic members of this regulon were significantly less abundant in the hmgA mutant. Taken together, the results suggest that the pyomelanization of $V$. campbellii sufficiently impairs the metabolic activities of this organism and renders it less fit and virulent than its isogenic wild type strain.

Keywords: Vibrio, melanin, bioluminescence, quorum sensing, tyrosine catabolism

\section{INTRODUCTION}

As a member of the L-tyrosine catabolism pathway in bacterial and eukaryotic organisms, the enzyme homogentisate 1,2dioxygenase (HmgA) catalyzes the intermediate homogentisic acid into 4-maleylacetoacetate which is further catabolized to yield fumarate and acetoacetate. In some bacterial species, it has been demonstrated that the inactivation of the $h m g A$ gene results in the accumulation of homogentisic acid which when autooxidized leads to the formation of the water-soluble brown pigment pyomelanin (Rodriguez-Rojas et al., 2009; Schmaler-Ripcke et al., 2009; Turick et al., 2009; Valeru et al., 2009; Wang et al., 2011). This phenotype has been observed in naturally pigmented environmental and clinical strains of Vibrio cholerae and has been shown to be due to mutations in the hmgA gene (Wang et al., 2011). Interestingly, pyomelanin pigmented V. cholerae demonstrate greater UV and oxidative stress resistance, virulence factor expression and infant mouse intestine colonization rates than their non-pigmented counterparts (Valeru et al., 2009). The ability of pyomelanin to confer increased resistance to oxidative stress appears to contribute to virulence by reducing the susceptibility of pigmented bacteria to host defense mechanisms. Because of these particular characteristics, it is not surprising that pyomelaninproducing Pseudomonas aeruginosa and Burkholderia cepacia are frequently isolated from cystic fibrosis patients (Zughaier et al., 1999; Rodriguez-Rojas et al., 2009). Furthermore, the production of pyomelanin has also been shown to provide greater protection from other environmental stresses such as hyperosmotic shock and elevated temperatures (Kotob et al., 1995) and act as a sole terminal electron acceptor and soluble electron shuttle to iron which may provide an additional fitness advantage to pyomelanin-producing mutants in anaerobic environments (Turick et al., 2002).

Despite these seemingly advantageous phenotypes, such pigmented mutants have not been reported from pathogenic members of the Vibrio Harveyi clade. Two of the most economically important Harveyi clade species, V. campbellii and V. harveyi, are common inhabitants of tropical marine environments and are among the most important bacterial pathogens of many 
commercially farmed marine invertebrate and vertebrate species (Thompson et al., 2004; Austin and Zhang, 2006). As certain pathogenic members of both species are capable of producing quorum sensing induced bioluminescence, the disease caused by them is often referred to as luminescent vibriosis (Defoirdt et al., 2008) and is a disease manifestation that is frequently implicated in outbreaks within penaeid shrimp larval culture facilities worldwide (Austin and Zhang, 2006). Given the importance of shrimp hemocyte-mediated oxidative defense mechanisms in combatting Vibrio infections (Ji et al., 2011), it is not unreasonable to posit that pyomelanization may benefit the survival and perhaps exacerbate the virulence of vibrios in this host environment. However, the production of pyomelanin comes at the cost of impairing the tyrosine catabolism pathway and the effect of the inactivation of $h m g A$ and/or pyomelanin production on global cellular metabolism is not known. In this study, we used V. campbellii ATCC BAA-1116, a bioluminescent marine bacterium that is best known as a model organism for quorum sensing studies (Bassler, 1999), to begin to determine the generality of pyomelaninmediated phenotypes and how the deletion of the $h m g A$ gene and resulting pyomelanin production may affect cellular phenotypes, virulence, and transcription.

\section{MATERIALS AND METHODS BACTERIAL STRAINS AND GROWTH CONDITIONS}

V. campbellii (ATCC strain BAA-1116; previously known as $V$. harveyi BAA-1116 or BB120; Lin et al., 2010) and the $h m g A$ mutant were grown in Luria Marine (LM) medium $(20 \mathrm{~g} \mathrm{NaCl}$, 10 tryptone, 5 g yeast extract per L, pH 7.8) or Tryptic Soy Broth containing $1 \% \mathrm{NaCl}$. Escherichia coli DH5 $\alpha$ and SM10 $\lambda$ pir used for standard DNA manipulation and conjugation were grown in Luria Broth (LB) medium.

\section{CONSTRUCTION OF THE hmgA IN-FRAME DELETION MUTANT}

The in-frame deletion of the $V$. campbellii hmgA gene $(\triangle h m g A)$ was generated by overlap PCR (Warrens et al., 1997). Briefly, $\sim 500$ bp DNA fragments upstream and downstream of the $h m g A$ open reading frame were amplified from V. campbellii BAA-1116 genomic DNA using the primer pairs $h m g A-\mathrm{a}\left(5^{\prime}-\right.$ TAggatccTGTACGAAATCGACCATCTGAC)/hmgA-b $\left(5^{\prime}-\mathrm{c}\right)$ and hmgA-c (5'-GAGGAGTACTAAGCGGGGGCAAGGATGAAA)/ hmgA-d (5'-CActcgagACTTCACCTTCGAAGTCAATCC), respectively. The two PCR products were annealed using their overlapping region and amplified using primers $h m g A-\mathrm{a}$ and $h m g A$-d. The resulting $1 \mathrm{~Kb}$ PCR fragment was cloned into the pCR4-TOPO vector using the TOPO TA cloning kit (Invitrogen, Carlsbad, CA, USA). This assembled fragment was then digested from the TOPO vector with BamHI and XhoI and cloned into the plasmid pZW125, which was constructed by inserting a chloramphenicol resistance gene into the oriR $\mathrm{R}_{R 6 \mathrm{Kg}}$ plasmid pWM91 containing the $s a c B$ gene (Metcalf et al., 1996). The resulting plasmid (pZW025) was transformed into E. coli strain Sm10 $\lambda$ pir and transferred into a V. campbellii spontaneous streptomycin resistant mutant ( $V$. campbellii-str1) by conjugation. The conjugants were grown on LM agar plates containing $3 \mu \mathrm{g} / \mathrm{mL}$ chloramphenicol and $1 \mu \mathrm{g} / \mathrm{mL}$ streptomycin. The $\Delta h m g A$ was selected on LM plates supplemented with $6 \%$ sucrose and verified by PCR.

\section{GROWTH CURVE ANALYSES}

Bacterial replication was measured using a Bioscreen $\mathrm{C}$ analyzer (Growth Curves USA, Piscataway, NJ, USA). Briefly, overnight cultures were diluted 1:5000 $\left(\sim 10^{5}\right.$ cells $\left./ \mathrm{mL}\right)$ in pre-warmed LM and five $200 \mu \mathrm{L}$ aliquots of the wild type (WT) and $\Delta h m g A$ strains were transferred into a 100 -well honeycomb plate. The plate was incubated at $30^{\circ} \mathrm{C}$ for $48 \mathrm{~h}$ with continuous shaking and wide band $\mathrm{OD}_{450-580 \mathrm{~nm}}$ measurements taken every $30 \mathrm{~min}$. Three independent experiments were performed in this manner.

\section{MEASUREMENT OF PIGMENTATION, BIOLUMINESCENCE AND CELLULAR SUSCEPTIBILITY $\mathrm{TO}^{\mathrm{H}_{2} \mathrm{O}_{2}}$}

Matched diluted overnight WT and $\triangle h m g A$ cultures were used to inoculate $50 \mathrm{~mL}$ LM media in $250 \mathrm{~mL}$ flasks and incubated at $30^{\circ} \mathrm{C}$ and $200 \mathrm{rpm}$. Every $24 \mathrm{~h}$, three $100 \mu \mathrm{L}$ aliquots of culture were collected and bacterial cells were pelleted via centrifugation at $10,000 \times g$ for $5 \mathrm{~min}$. Supernatant pigments were measured using a NanoDrop ND-2000c spectrophotometer (Thermo Scientific, Pittsburg, PA, USA) at $\mathrm{OD}_{400}$. Another three $100 \mu \mathrm{L}$ aliquots of culture were placed in a black U96 Nunc MicroWell ${ }^{\mathrm{TM}}$ plate (Thermo Scientific) and measured for bioluminescence using a Luminoskan Ascent Microplate Luminometer (Thermo Scientific). Three independent experiments were performed in this manner.

At the $48 \mathrm{~h}$ time point, WT, and $\Delta h m g A$ cells were harvested, washed and resuspended in fresh LM media. They were then incubated with $2 \mathrm{mM} \mathrm{H}_{2} \mathrm{O}_{2}$ at room temperature for $15 \mathrm{~min}$. The percentage survival was calculated by counting colony forming units (CFU) immediately before and after the $\mathrm{H}_{2} \mathrm{O}_{2}$ treatment on LM agar plates. The potentially protective effect of WT and $\Delta h m g A$ culture supernatants against $\mathrm{H}_{2} \mathrm{O}_{2}$ treatment was also tested using WT cells. Briefly, mid-log phase WT cells were harvested, washed and resuspended in $0.2 \mu \mathrm{m}$ filter-sterilized supernatants from WT and $\Delta h m g A 48 \mathrm{~h} \mathrm{LM}$ media cultures. The cell suspensions were then incubated at room temperature in the presence of $2 \mathrm{mM} \mathrm{H}_{2} \mathrm{O}_{2}$ for $15 \mathrm{~min}$. The percentage survival was calculated by counting CFUs immediately before and after the $\mathrm{H}_{2} \mathrm{O}_{2}$ treatment on LM agar plates. The data for each of these experiments was generated from three independent assays.

\section{PIGMENT PREPARATION AND ELECTRON SPIN RESONANCE SPECTROSCOPY}

Partial purification of the pigment from the $\Delta h m g A$ strain was modified from the method previously described by Turick et al. (2002). Briefly, a $50 \mathrm{~mL} \Delta h m g A$ culture was grown in LM at $30^{\circ} \mathrm{C}$ for $96 \mathrm{~h}$ with shaking at $200 \mathrm{rpm}$. The cells were harvested via centrifugation at $5000 \times g$ for $10 \mathrm{~min}$, and the supernatant was removed and acidified with $6 \mathrm{~N} \mathrm{HCl}$ to a final solution concentration of $0.4 \mathrm{~N}$ and was then allowed to precipitate for $12 \mathrm{~h}$ at room temperature. The concentrated pigment was collected by centrifugation at $8000 \times g$ for $20 \mathrm{~min}$, washed twice with $\mathrm{dH}_{2} \mathrm{O}$ and then dried using a SpeedVac Concentrator (Thermo Scientific). A pure synthetic melanin that was chemically prepared from the oxidation of tyrosine was purchased and used as a control (M8631, Sigma-Aldrich, St. Louis, MO, USA). A second control, DHN-melanin, was prepared from the conidia of the fungus Aspergillus niger using the method of Youngchim et al. (2004). The pigment powder samples were characterized by electron 
spin resonance (ESR) at $300 \mathrm{~K}$ in a Bruker $9.5 \mathrm{GHz}$ spectrometer. Typical microwave powers of $5-20 \mathrm{~mW}$ with $1 \mathrm{G}$ modulation amplitude and $100 \mathrm{kHz}$ field modulation were employed for these experiments.

\section{SHRIMP VIRULENCE ASSAYS}

The $\mathrm{LD}_{50}$ of the WT and $\Delta h m g A$ strains were evaluated on the black tiger shrimp Penaeus monodon. Both strains were grown in Tryptic Soy Broth containing $1 \% \mathrm{NaCl}$ at $30^{\circ} \mathrm{C}$ with shaking at $150 \mathrm{rpm}$, harvested by centrifugation at $2000 \times g$ for $10 \mathrm{~min}$ and washed twice with sterile Marinum ${ }^{\circledR}$ artificial seawater (ASW) (Mariscience International Co. Ltd., Bangkok, Thailand). Bacterial cell suspensions in ASW were adjusted to $2.6 \times 10^{8} \mathrm{CFU} / \mathrm{mL}$ using a turbidimeter (Oxoid Ltd., United Kingdom) and twofold dilutions were performed to obtain the required concentrations of bacteria prior to injecting the shrimp. The juvenile shrimp used in this study were $10-13 \mathrm{~g}$ in weight and 4-5 inches in length. Each shrimp received an intramuscular injection of $100 \mu \mathrm{L}$ diluted $V$. campbellii (with batches of seven shrimp/dose) between the third and fourth abdominal segments. Control shrimp were injected with ASW. The experiments were performed in quadruplicate. The animals were maintained in a $70 \mathrm{~L}$ ASW glass tank at a temperature of $29 \pm 1^{\circ} \mathrm{C}$ and salinity of 17 ppt. Shrimp mortalities were observed within $48 \mathrm{~h}$ of injection and were confirmed by detecting bioluminescence in the organs of the dead shrimp. The $\mathrm{LD}_{50}$ was calculated using the method of Reed and Muench (1938).

\section{MICROARRAY-BASED TRANSCRIPTOME ANALYSES}

Aliquots of three cultures $\left(3.0 \times 10^{8}\right.$ cells $\left./ \mathrm{mL}\right)$ of the WT and $\triangle h m g A$ strains grown in $\mathrm{LM}$ at $30^{\circ} \mathrm{C}$ for $48 \mathrm{~h}$ with constant shaking at $200 \mathrm{rpm}$ were harvested for total RNA extraction. RNA was isolated using the RiboPure ${ }^{\mathrm{TM}}-$ Bacteria Kit (Life Technologies, Grand Island, NY, USA), treated with DNase and $10 \mu \mathrm{g}$ of total RNA from each culture was further purified using the MICROBExpress ${ }^{\mathrm{TM}}$ Bacterial mRNA Enrichment Kit (Life Technologies) according to the manufacturer's specifications. All RNA preparations were quantified and analyzed using the Agilent 2100 Bioanalyzer (Agilent Technologies, Inc., Santa Clara, CA, USA) and normalized to $1 \mu \mathrm{g}$. The normalized RNA was labeled, purified, fragmented and hybridized to a custom Affymetrix microarray (520694F) according to standard protocols (Affymetrix, Santa Clara, CA, USA). All hybridizations incubated for $16 \mathrm{~h}$ at $49^{\circ} \mathrm{C}$ in the GeneChip ${ }^{\circledR}$ Hybridization Oven 640 at $60 \mathrm{rpm}$ and the microarrays were then washed and stained with the GeneChip ${ }^{\circledR}$ Fluidics Station 450 and scanned using the GeneChip ${ }^{\circledR}$ Scanner 7G (Affymetrix). Hybridization signal intensities were analyzed with the GeneChip ${ }^{\circledR}$ Operating Software (GCOS) to generate raw image files (.DAT) and simplified image files (.CEL) with intensities assigned to each of the corresponding probe positions. The data collected was used to profile the expression levels of 4831 open reading frames. The data containing the distribution of the probe amplitudes were calculated and a classical analysis of variance (ANOVA; applying the CRAN R's aov function) was performed across conditions for each probe site. The median values revealing the gene-level measurement of differential expression was determined (Rubin, 2009). The gene designations and annotations utilized are from the Naval Research Laboratory's $V$. campbellii ATCC BAA-1116 genome sequencing effort (GenBank accession numbers CP006605, CP006606, CP006607) and the expression profiling data can be found in the GenBank Gene Expression Omnibus repository (accession number GSE46223).

\section{QUANTITATIVE REVERSE TRANSCRIPTION PCR}

Real-time reverse transcription PCR assays were conducted using the iScript ${ }^{\mathrm{TM}}$ One-Step RT-PCR Kit with SYBR Green (Bio-Rad Laboratories, Hercules, CA, USA). One nanogram of mRNA from two biological replicates were tested in triplicate on an iCycler (BioRad). The PCR primers were designed using Primer3 online software (v. 0.4.0) (http://frodo.wi.mit.edu/). Relative quantities of the transcripts were determined using the $2^{-\Delta \Delta} \mathrm{Ct}$ formula where $\Delta \mathrm{Ct}$ is the difference in $\mathrm{Ct}$ of the selected genes and $\mathrm{Ct}$ of the normalizer gene, and $\Delta \Delta \mathrm{Ct}$ is the difference in $\Delta \mathrm{Ct}$ from $\Delta h m g A$ and $\Delta \mathrm{Ct}$ from the WT. The rpoS1 gene was used to normalize the expression levels of the selected genes as its transcription level was found to be constant in both the WT and $\Delta h m g A$ strains.

\section{RESULTS AND DISCUSSION CHARACTERIZATION OF $\Delta$ hmgA PIGMENT AND PHENOTYPES}

An examination of the $V$. campbellii ATCC BAA-1116 genome revealed that 4 genes in the catabolic pathway of tyrosine metabolism, hmgA (M892_02450), hppd (4hydroxyphenylpyruvate dioxygenase, M892_02455), fahA (fumarylacetoacetase, M892_02445), and maiA (maleylacetoacetate isomerase, M892_02440), appeared to form an operon and shared the same genetic synteny as other sequenced Vibrio species. An in-frame deletion of the $h m g A$ gene was generated $(\Delta h m g A)$ to investigate the role of this gene in pigment production and cellular physiology. When cultured on LM agar plates, in baffled glass Erlenmeyer flasks or polystyrene round-bottomed tubes for $\geq 48 \mathrm{~h}$, the $\Delta h m g A$ strain produced a brown pigment that was expected to be the result of the auto-oxidation and polymerization of homogentisate (Figures 1A,B). The pigment was found to be present in $\triangle h m g A$ cell-free supernatants as well as washed cell pellets suggesting that the pigment was not only released into the microenvironment but could also be found associated with the bacterial cell membrane (data not shown). Interestingly, the production of this pigmentation was not observed when $\Delta h m g A$ cells were cultured in $50 \mathrm{~mL}$ polypropylene conical tubes (Figure 1C). Under the experimental conditions utilized, the $50 \mathrm{~mL}$ conical tubes provided the least aeration. In the absence of sufficient aeration, which is required for the oxidation of homogentisate, the production of the pigment was not observed. In order to identify the oxidized homogentisate polymer, the extracted brown pigment from $\triangle h m g A$ cell culture supernatants was examined using ESR. The ESR spectrum of the pigment revealed a distinct stable free radical signal that was characteristic of melanin and highly similar to synthetic eumelanin and DHN melanin of Aspergillus niger (Figure 1D) thus, confirming that the $\Delta h m g A$ pigment was pyomelanin. The results demonstrate that like $V$. cholerae 

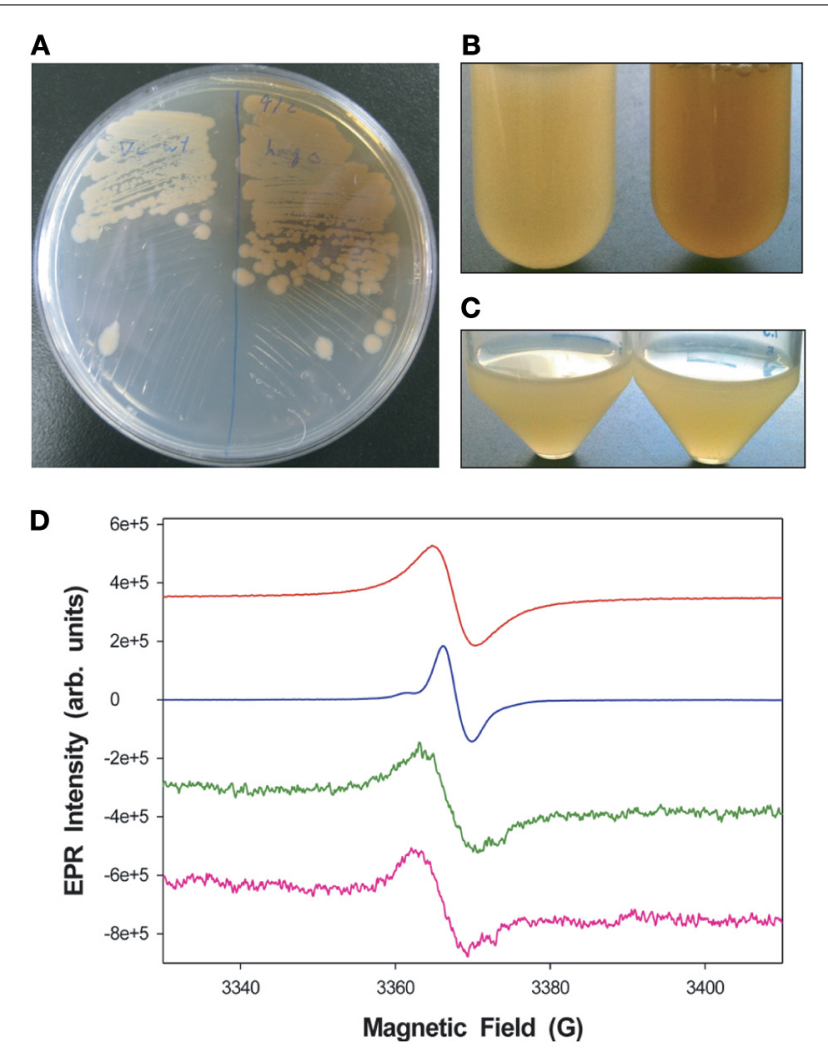

FIGURE 1 | Pigmentation differences between $\boldsymbol{V}$. campbellii WT and $\boldsymbol{\Delta} \boldsymbol{h m g} \boldsymbol{A}$. (A) Colonies grown on LM agar. WT (left), $\Delta h m g A$ (right); (B) Cel cultures grown in LM in borosilicate glass tubes (48 h). WT (left), $\Delta h m g A$ (right); (C) Cell cultures grown in LM in polypropylene conical tubes (48 h). WT (left), $\Delta$ hmgA (right); (D) EPR spectra of synthetic melanin (red), $\mathrm{DHN}$-melanin (blue) and partially purified pigments from the supernatants of two independent $\Delta h m g A$ cultures (green and pink).

$\Delta h m g A, V$. campbellii $\Delta h m g A$ also produces pyomelanin but does not appear to do so in the same abundance or nearly as rapidly (Valeru et al., 2009).

Growth curve analyses in nutrient rich LM medium revealed that the WT and $\Delta h m g A$ strains grew equally well during the lag, log, and early stationary phases of growth. However, $\Delta h m g A$ displayed a lesser ability to survive during late stationary phase (post $48 \mathrm{~h}$ ) (Figure 2A): a time point that coincided with the measurable production of pyomelanin (Figure $2 \mathrm{~B}$ ).

In other bacterial and fungal organisms, melanin has been demonstrated to have a role in protecting against certain environmental stressors as mutations in the $h m g A$ gene and the resulting melanization have been shown to provide an increased resistance to UV irradiation and $\mathrm{H}_{2} \mathrm{O}_{2}$-mediated oxidative stress (Rodriguez-Rojas et al., 2009; Schmaler-Ripcke et al., 2009; Valeru et al., 2009). However, when we performed similar experiments comparing the response of $V$. campbellii WT and $\Delta h m g A$ washed cells to different doses of UV irradiation and concentrations of $\mathrm{H}_{2} \mathrm{O}_{2}$, no significant differences in viability were observed (data not shown). Interestingly, however, we did observe the protective
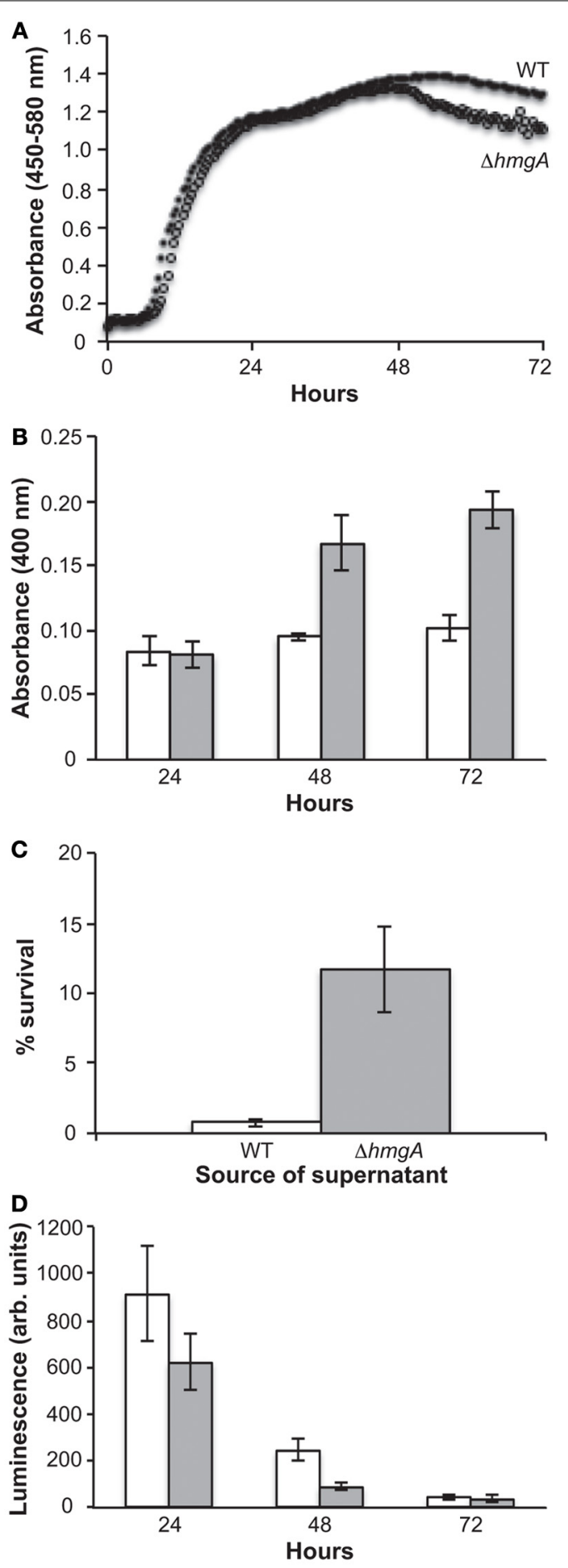

FIGURE 2 | Growth, pigment production and bioluminescence in V. campbellii WT and $\boldsymbol{\Delta} \boldsymbol{h} \boldsymbol{m g} \boldsymbol{A}$. (A) Grow curves of the WT and $\Delta h m g A$ strains in LM at $30^{\circ} \mathrm{C}$. WT (closed circle), $\Delta h m g A$ (open circle); (B) Absorbance of pigment-containing supernatants. WT (white bars), $\Delta h m g A$ (gray bars); (C) Survival of WT V. campbellii cells in $2 \mathrm{mM} \mathrm{H}_{2} \mathrm{O}_{2}$ when resuspended in supernatants from WT or $\Delta h m g A$ cultures; (D) Bioluminescence measurements. WT (white bars), $\Delta h m g A$ (gray bars). Error bars represent the standard deviation from three experiments. 
properties of pyomelanin when cell-free pyomelanin-containing supernatants from $\Delta h m g A$ cultures were incubated with WT cells and then exposed to $2 \mathrm{mM} \mathrm{H}_{2} \mathrm{O}_{2}$ (Figure 2C). Comparatively, cell-free supernatants from WT cultures did not demonstrate this protective function. The combined $\mathrm{H}_{2} \mathrm{O}_{2}$ challenge results demonstrated the protective material property of $V$. campbellii pyomelanin and suggest that there is not sufficient membraneassociated pyomelanin in $V$. campbellii to afford this same level of protection in the absence of the accumulated extracellular pyomelanin.

Unlike any other pyomelanin-producing bacterium investigated to date, V. campbellii ATCC BAA-1116 is capable of generating quorum sensing-induced bioluminescence. Interestingly, previous studies have suggested that the luciferase enzyme and bacterial bioluminescence, like pyomelanin, also play a physiological role in protecting cells against UV and oxidative stress. For example, UV irradiation has been found to stimulate bioluminescence (Czyz et al., 2002) which in turn photoreactivates DNA repair processes (Kozakiewicz et al., 2005) and bioluminescent cells have been shown to be significantly more resistant to UV irradiation than their non-bioluminescent counterparts (Czyz et al., 2000; Kozakiewicz et al., 2005). In addition, various oxidants, such as $\mathrm{H}_{2} \mathrm{O}_{2}$, have been shown to severely impair the growth of vibrios lacking the luciferase enzyme (Szpilewska et al., 2003). This is due to the fact that in addition to the production of light, this enzyme is also capable of increasing cellular resistance to oxidative stress by detoxifying reactive oxygen species (Katsev et al., 2004). Therefore, in addition to the more common bacterial mechanisms of UV and oxidative stress protection, bioluminescent $V$. campbellii also contain luciferasebased protective mechanisms. This combination of luciferase and pyomelanin in the same organism introduced the possibility of additive or synergistic stress protection. However, when the bioluminescence output of $\Delta h m g A$ was examined during measurable pyomelanin production $(48 \mathrm{~h})$, it was found to be significantly attenuated in comparison to the WT (Figure 2D). While this result demonstrated a diminution of light production in pyomelanin-producing $\Delta h m g A$, it was incapable of determining whether this was due to a decrease in luciferase abundance or activity, reduced intracellular $\mathrm{O}_{2}$ or necessary substrates.

\section{SHRIMP VIRULENCE MODEL}

In $V$. cholerae $\triangle h m g A$ and $P$. aeruginosa $\Delta h m g A$, pyomelanization was shown to play a role in increasing virulence factor expression and adaptation to chronic infections in vertebrate animal models (Rodriguez-Rojas et al., 2009; Valeru et al., 2009). As V. campbellii BAA-1116 is known to pathogenize shrimp, we sought to determine whether V. campbellii pyomelanization would have a similar effect on invertebrate animal infections. V. campbellii WT and $\Delta h m g A$ were used to infect juvenile black tiger shrimp (Penaeus monodon) and the $\mathrm{LD}_{50}$ of both were evaluated. Surprisingly, the $\mathrm{LD}_{50}$ of $\Delta h m g A$ was $\sim 2.7$ times higher (less lethal) than that of the WT indicating that the production of pyomelanin in $\Delta h m g A$ was associated with decreased virulence in this model infection system (Table $\mathbf{1}$ ).

In invertebrates, one of two major immune responses against invading pathogens is the prophenoloxidase activating system
Table 1 | V. campbellii BAA-1116 and $\Delta$ hmgA Penaeus monodon challenge.

\begin{tabular}{lcc}
\hline Experiment no. & \multicolumn{2}{c}{ LD $_{\mathbf{5 0}}$ (CFU/mL) } \\
\cline { 2 - 3 } & $\mathbf{W T}$ & $\boldsymbol{\Delta h} \mathbf{h m A}$ \\
\hline 1 & $1.4 \times 10^{8}$ & $>3.1 \times 10^{8}$ \\
2 & $2.1 \times 10^{8}$ & $4.1 \times 10^{8}$ \\
3 & $2.4 \times 10^{8}$ & $5.8 \times 10^{8}$ \\
4 & $1.9 \times 10^{8}$ & $5.4 \times 10^{8}$ \\
Mean \pm SD & $1.9 \pm 0.4 \times 10^{8}$ & $5.1 \pm 0.9 \times 10^{8}$
\end{tabular}

Four independent $L D_{50}$ experiments were performed. Mean values of $L D_{50}$ between the WT and $\Delta h m g A$ were significantly different $(P<0.05$, student's t-test).

(proPO) (Cerenius and Soderhall, 2004). Upon infection, nonself molecules such as lipopolysaccharide, peptidoglycan and $\beta$-glucan can activate the proPO I cascade and result in the formation of melanin around the invading microorganisms. In this circumstance, the host formed melanin is thought to physically shield the pathogens to prevent or retard their growth. Host derived quinones, which are intermediates of melanin production, may also be involved in the production of cytotoxic molecules (e.g., superoxides, hydroxyl radicals) that could help inactivate the invading pathogens. The protective efficacy of the proPO system is further highlighted by the demonstration that gene silencing of PO activating enzymes in Penaeus monodon increases the susceptibility of the host to $V$. harveyi infection (Amparyup et al., 2009; Charoensapsri et al., 2009). Given these facts and the demonstrated phenotypes of $V$. campbellii $\Delta h m g A$, we suggest that pyomelanization may reduce this bacterium's virulence potential in two ways. First, pyomelanin production by $\triangle h m g A$ cells may effectively add another layer to the host-assembled melanin around the sites of infection so as to further limit bacterial growth. The comparatively poor survival of $\triangle h m g A$ in late stationary phase (the time of melanization) (Figure 2A) may allude to this possibility. Second, the pyomelanin produced by $\Delta h m g A$ may be recognized by the host as another foreign moiety that could further stimulate the proPO system and enhance the clearance of these bacteria.

\section{TRANSCRIPTOME ANALYSES}

As the differences in pyomelanin production, bioluminescence and survival were observed during late stationary phase $(48 \mathrm{~h})$, we chose this time point to perform comparative microarray-based expression profiling analyses to understand how the deletion of the $h m g A$ gene and subsequent production of pyomelanin gave rise to the observed phenotypes. Whole genome expression profiling revealed that 129 genes ( $2.7 \%$ of the interrogated genome) were significantly modulated in $\triangle h m g A$ when compared to the WT (adjusted $p$-value $<0.001$ ). Overall, inactivation of the $h m g A$ gene appeared to affect the expression of genes involved in energy production and conversion, amino acid metabolism, lipid metabolism, and quorum sensing/bioluminescence (Figure 3). Interestingly, the transcript levels of all 129 genes were found to be less abundant in $\triangle h m g A$ with approximately $70 \%$ of them 


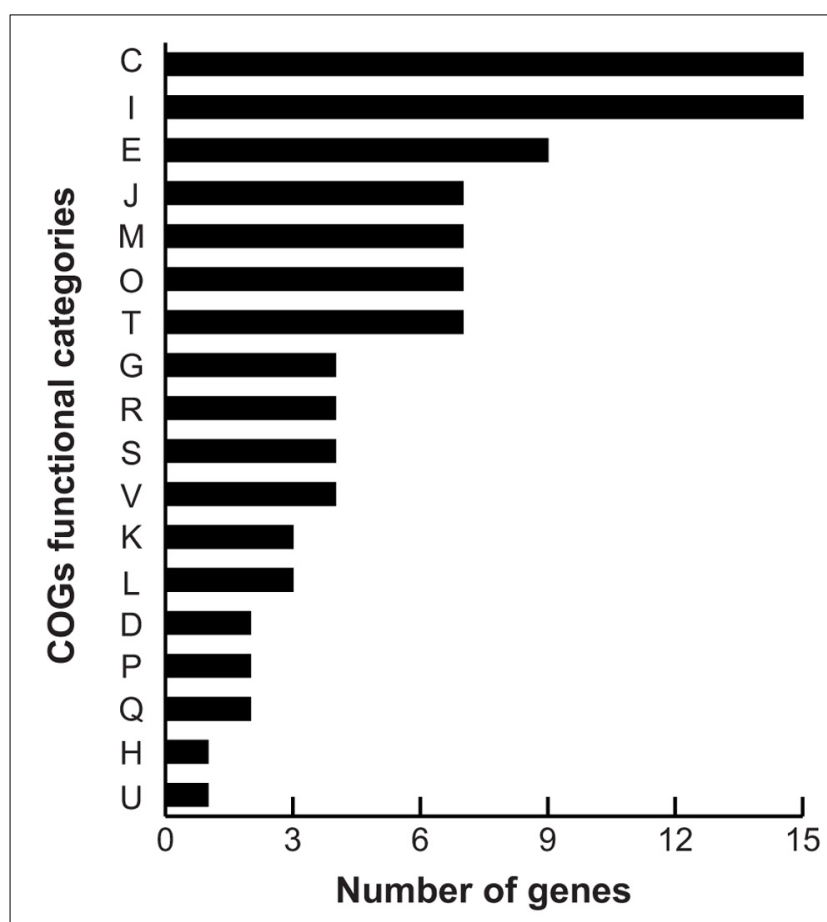

FIGURE 3 | COG summary of differentially expressed genes. COG functional category: $C$, energy production and conversion; I, lipid transport and metabolism; E, amino acid transport and metabolism; J, translation, ribosomal structure and biogenesis; $M$, cell wall/membrane/envelope biogenesis; O, post-translational modification, protein turnover, chaperones; T, signal transduction mechanisms; $\mathrm{G}$, carbohydrate transport and metabolism; R, general function prediction only; $S$, function unknown; $V$, defense mechanisms; $K$, transcription; $L$, replication, recombination and repair; $D$, cell cycle control, cell division, chromosome partitioning; $P$, inorganic ion transport and metabolism; Q, secondary metabolites biosynthesis, transport and catabolism; $\mathrm{H}$, coenzyme transport and metabolism; $U$, intracellular trafficking, secretion, and vesicular transport.

demonstrating $\mathrm{a} \geq$ twofold reduction in transcript abundance (Table 2). The transcriptional modulation of 10 of these genes was also verified using quantitative RT-PCR (Table 2).

\section{ENERGY PRODUCTION AND ELECTRON TRANSFER}

Two of 3 genes (M892_08460, M892_08470) in an operon encoding subunits of ubiquinol-cytochrome $c$ reductase were down-regulated in $\triangle h m g A$. This cytochrome complex catalyzes the oxidoreduction of mobile redox components generating an electrochemical potential (Kurowski and Ludwig, 1987). Two other modulated genes, NrfB (M892_17240) and NrfC (M892_17245), encode cytochrome-type components of the electron transfer chain of respiratory nitrite ammonification in $\gamma$ proteobacteria (Simon, 2002). This electron transfer reaction usually occurs during anaerobic growth and the electron donor formate is readily formed from pyruvate by pyruvate formate lyase. Coincidently, the gene encoding pyruvate formate lyase (M892_05385) was also repressed in the mutant. In addition, a number of genes in the glycolysis pathway, such as those encoding fructose-bisphosphate aldolase (M892_13305), glyceraldehyde 3-phosphate dehydrogenase (M892_15785) and enolase
(M892_19270), were down-regulated as well, and likely result in the reduced production of pyruvate. Since HmgA is not a DNA regulatory factor, these results indicated that the pyomelanin produced in $\triangle h m g A$ may decrease redox activity by serving as an electron sink which in turn would interfere with electron transfer and further weaken cellular respiration in late stationary phase.

\section{AMINO ACID AND LIPID METABOLISM}

The transcripts from three well characterized gene clusters involved in amino acid and lipid metabolism (etfABD, ivd$A B C D E F G$, liuABCDE) were also significantly down-regulated in $\triangle h m g A$. These operons encode enzymes that take part in the branched-chain amino acid (i.e., isoleucine, leucine, valine) degradation pathway that is used for energy production in many proteobacteria (Kazakov et al., 2009). Proteins encoded by etfABD catalyze electron transfer from quinones to flavoproteins not only in the leucine degradation pathway but also in the fatty acid degradation pathway. The master regulator of the branched-chain amino acid degradation pathway, LiuR, is encoded by the liuR gene which resides between the liuABCDE and ivdABCDEFG operons. The $l i u R$ gene was also significantly down-regulated in $\Delta h m g A$ ( $p$-value $=0.0003)$ suggesting a regulatory mechanism for the pyomelanin-induced retardation of branched-chain amino acid degradation.

It was interesting to note that another amino acid degradation pathway was also affected by the production of pyomelanin. The proline utilization operon putBCP, encoding proline dehydrogenase, $\delta$-1-pyroline-5-carboxylate dehydrogenase and permease, respectively, was also down-regulated in $\Delta h m g A$. However, the expression of $p r u R$, which is adjacent to and has been reported to regulate the $p u t B C P$ operon in $P$. aeruginosa PAO1 (Nakada et al., 2002), did not change. Interestingly, transcription of the putBCP operon has been reported to respond to osmotic stress by producing more of the final product glutamate in $\mathrm{V}$. vulnificus (Lee et al., 2003) and coincidently, pyomelanin biosynthesis was also demonstrated to be induced by osmotic stress in V. cholerae (Coyne and Al-Harthi, 1992). Thus, it is possible that $\Delta h m g A$ pyomelanin may function as a solute glutamate to counter osmotic stress during late stationary phase thus, alleviating the physiological signal for the increased expression of $p u t B C P$ and resulting in the decreased transcription observed in this study.

\section{VIRULENCE, QUORUM SENSING, AND BIOLUMINESCENCE}

Although the virulence mechanisms that contribute to bioluminescent vibriosis are not completely understood, several biomolecules are known to be contributing factors (Austin and Zhang, 2006). The expression of many of these virulence factors, including the type III secretion system (Henke and Bassler, 2004a), extracellular toxin (Manefield et al., 2000), metalloprotease (Mok et al., 2003), siderophore (Lilley and Bassler, 2000), chitinase (Defoirdt et al., 2010), phospholipase, caseinase, and gelatinase (Natrah et al., 2011) are known to be regulated by the quorum sensing master regulator LuxR. Our findings from the shrimp virulence model demonstrated that $\Delta h m g A$ was less virulent than the WT and led to the suggestion that this phenotype may have been due to the down-regulation of 
Table 2 | Differentially expressed genes in $\Delta h m g A$ compared with WT $V$. campbellii.

\begin{tabular}{|c|c|c|c|c|c|c|c|}
\hline Gene ID & Annotation & pval & $\mathbf{W T}^{\mathbf{a}}$ & $\Delta h m g A$ & Change $^{b}$ & qRT-PCR ${ }^{c}$ & Function $^{e}$ \\
\hline M892_06250 & 2-oxoglutarate dehydrogenase & $5.20 \mathrm{E}-04$ & 1.29 & 0.67 & 1.93 & & $\mathrm{C}$ \\
\hline M892_06275 & Citrate synthase & 1.57E-06 & 2.31 & 0.72 & 3.21 & & $\mathrm{C}$ \\
\hline M892_08460 & Ubiquinol-cytochrome c reductase & 1.12E-05 & 1.45 & 0.61 & 2.38 & & $\mathrm{C}$ \\
\hline M892_13885 & Aconitate hydratase & $2.42 \mathrm{E}-06$ & 2.21 & 1.12 & 1.97 & & $\mathrm{C}$ \\
\hline M892_17240 & Cytochrome c-type protein NrfB & 1.17E-06 & 1.24 & 0.04 & 31.00 & & $\mathrm{C}$ \\
\hline M892_17245 & Nitrite reductase, Fe-S protein NrfC & 1.73E-06 & 1.24 & 0.02 & 62.00 & 1.58 & $\mathrm{C}$ \\
\hline M892_23815 & acyl-CoA dehydrogenase, short-chain specific EtfA & $6.43 E-06$ & 1.72 & 0.52 & 3.31 & 5.30 & $\mathrm{C}$ \\
\hline M892_23820 & Electron transfer flavoprotein subunit beta EtfB & 5.49E-05 & 1.69 & 0.73 & 2.32 & & $\mathrm{C}$ \\
\hline M892_08365 & Cell division protein FtsA & $3.06 \mathrm{E}-04$ & 1.13 & 0.79 & 1.43 & & $\mathrm{D}$ \\
\hline M892_03740 & Aspartate aminotransferase & $2.04 \mathrm{E}-04$ & 0.90 & 0.32 & 2.81 & & $\mathrm{E}$ \\
\hline M892_03990 & Phosphoribosyl-AMP cyclohydrolase & $2.70 \mathrm{E}-05$ & 1.57 & 0.87 & 1.80 & & $\mathrm{E}$ \\
\hline M892_03995 & Imidazoleglycerol-phosphate synthase & 4.98E-04 & 1.51 & 1.04 & 1.45 & & $E$ \\
\hline M892_12425 & Argininosuccinate lyase & 1.13E-06 & 1.94 & 1.00 & 1.94 & & $E$ \\
\hline M892_13555 & Aspartokinase & $2.04 \mathrm{E}-04$ & 0.92 & 0.07 & 13.14 & & $E$ \\
\hline M892_16070 & Lactoylglutathione lyase & $5.98 \mathrm{E}-08$ & 3.09 & 2.02 & 1.53 & & $\mathrm{E}$ \\
\hline M892_27505 & Sodium/proline symporter PutP & 1.13E-06 & 2.02 & 0.78 & 2.59 & & $E$ \\
\hline M892_27510 & Delta 1-pyrroline-5-carboxylate dehydrogenase PutC & $5.98 \mathrm{E}-08$ & 3.20 & 1.87 & 1.71 & 4.18 & $E$ \\
\hline M892_27515 & Proline dehydrogenase PutB & 2.10E-07 & 3.04 & 1.27 & 2.39 & & $\mathrm{E}$ \\
\hline M892_24040 & 3-hydroxyisobutyrate dehydrogenase IvdF & $5.61 \mathrm{E}-06$ & 1.73 & 0.70 & 2.47 & & I \\
\hline M892_24045 & Enoyl-CoA hydratase/isomerase IvdE & $9.29 \mathrm{E}-06$ & 2.06 & 0.66 & 3.12 & & I \\
\hline M892_24050 & 3-hydroxyisobutyryl-CoA hydrolase IvdD & $2.15 \mathrm{E}-05$ & 1.73 & 0.36 & 4.81 & & I \\
\hline M892_24055 & Branched-chain acyl-CoA dehydrogenase IvdC & 1.59E-04 & 1.12 & 0.46 & 2.43 & & 1 \\
\hline M892_24060 & Methylmalonate-semialdehyde dehydrogenase IvdB & 1.49E-06 & 2.18 & 0.55 & 3.96 & 2.49 & I \\
\hline M892_24065 & 3-ketoacyl-CoA thiolase IvdA & $1.98 \mathrm{E}-05$ & 1.94 & 0.79 & 2.46 & & I \\
\hline M892_24075 & Isovaleryl-CoA dehydrogenase LiuA & $3.91 \mathrm{E}-06$ & 2.13 & 0.87 & 2.45 & & । \\
\hline M892_24080 & Methylcrotonyl-CoA carboxylase carboxyl transferase LiuB & $5.84 \mathrm{E}-05$ & 1.46 & 0.66 & 2.21 & & I \\
\hline M892_24525 & Polyhydroxyalkanoic acid synthase PhaC & 1.27E-05 & 1.05 & 0.10 & 10.50 & & 1 \\
\hline M892_24530 & PHA granule-associated protein PhaD & $5.48 \mathrm{E}-05$ & 3.07 & 0.57 & 5.39 & & । \\
\hline M892_24535 & Acetyl-CoA acetyltransferase PhaA & 4.29E-06 & 3.25 & 0.95 & 3.42 & 6.61 & I \\
\hline M892_24540 & Acetoacetyl-CoA reductase $\mathrm{PhaB}$ & $9.29 \mathrm{E}-06$ & 2.97 & 0.83 & 3.58 & & I \\
\hline M892_16425 & Acyl carrier protein & 8.93E-06 & 4.07 & 1.59 & 2.56 & & । \\
\hline M892_02880 & Ribosomal protein L35 & $5.66 \mathrm{E}-04$ & 2.01 & 1.40 & 1.44 & & J \\
\hline M892_02885 & Bacterial protein translation initiation factor 3 & 1.83E-05 & 2.95 & 2.39 & 1.23 & & J \\
\hline M892_07900 & Ribosome-associated protein $Y$ & $6.25 \mathrm{E}-05$ & 4.21 & 2.97 & 1.42 & & J \\
\hline M892_12355 & Translation elongation factor & $8.45 \mathrm{E}-04$ & 1.71 & 0.60 & 2.85 & & J \\
\hline M892_12355 & Translation elongation factor & $4.38 \mathrm{E}-07$ & 3.54 & 2.41 & 1.47 & & J \\
\hline M892_12355 & Translation elongation factor & $1.01 \mathrm{E}-05$ & 3.41 & 2.02 & 1.69 & & J \\
\hline
\end{tabular}


Table 2 | Continued

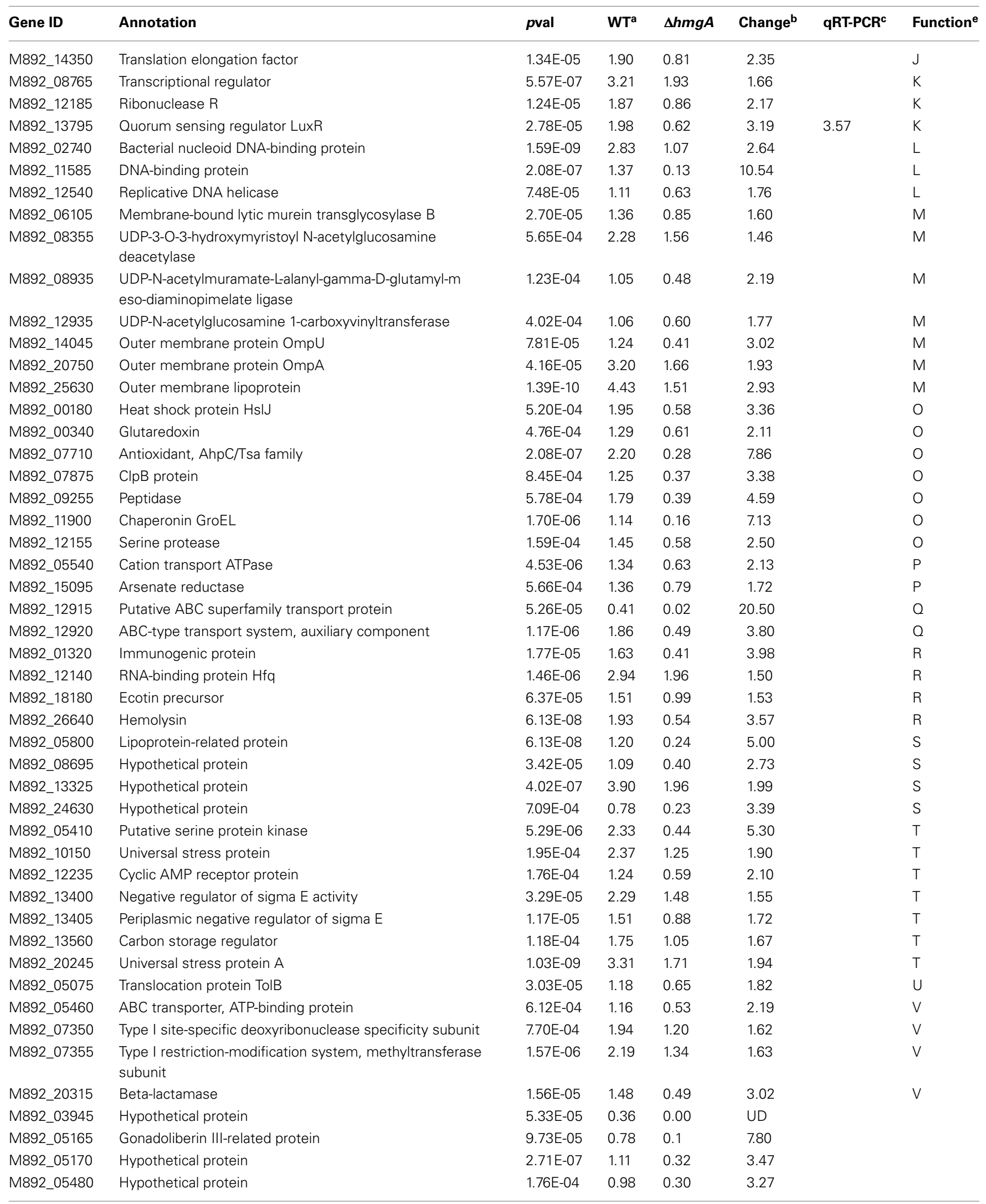

(Continued) 
Table 2 | Continued

\begin{tabular}{|c|c|c|c|c|c|c|c|}
\hline Gene ID & Annotation & pval & $\mathbf{W T}^{\mathbf{a}}$ & $\Delta h m g A$ & Change $^{b}$ & qRT-PCR ${ }^{c}$ & Function ${ }^{\mathrm{e}}$ \\
\hline M892_10485 & NADH dehydrogenase subunit II-related protein & 1.66E-08 & 3.75 & 2.87 & 1.31 & & \\
\hline M892_13365 & Hypothetical protein & 1.73E-04 & 3.25 & 1.17 & 2.78 & & \\
\hline M892_15830 & Hypothetical protein & 7.46E-05 & 1.24 & 0.30 & 4.13 & & \\
\hline M892_18970 & Hypothetical protein & $2.82 \mathrm{E}-05$ & 1.39 & 0.25 & 5.56 & & \\
\hline M892_19525 & Putative lipoprotein & 1.86E-08 & 4.17 & 1.09 & 3.83 & & \\
\hline M892_19565 & Muconate cycloisomerase I & $2.36 \mathrm{E}-04$ & 1.64 & 0.41 & 4.00 & & \\
\hline M892_19745 & Hypothetical protein & $3.75 \mathrm{E}-04$ & 0.39 & 0.00 & UD & & \\
\hline M892_20825 & Fatty acid reductase LuxC & 9.07E-06 & 2.64 & 1.23 & 2.15 & & \\
\hline M892_20850 & NAD(P)H-dependent FMN reductase LuxG & $4.58 \mathrm{E}-06$ & 1.78 & 0.81 & 2.20 & & \\
\hline M892_22630 & Peptidase & 1.03E-04 & 1.74 & 1.05 & 1.66 & & \\
\hline M892_22965 & Positive response regulator for Pho regulon & $3.60 \mathrm{E}-06$ & 2.16 & 0.82 & 2.63 & & \\
\hline M892_24070 & Transcriptional regulator LiuR & 0.0003 & 0.25 & 0.00 & UD & & \\
\hline M892_24215 & Putative GTPase & 4.14E-05 & 1.14 & 0.50 & 2.28 & & \\
\hline M892_24655 & Hypothetical protein & $3.22 \mathrm{E}-05$ & 4.11 & 1.48 & 2.78 & & \\
\hline M892_25115 & Transcriptional regulator LuxT & $2.58 \mathrm{E}-04$ & 2.96 & 2.25 & 1.32 & & \\
\hline M892_25430 & Azurin & 5.16E-04 & 1.88 & 1.20 & 1.57 & & \\
\hline M892_25755 & ATP-dependent protease & 7.73E-04 & 0.75 & 0.26 & 2.88 & & \\
\hline M892_25795 & Predicted transcriptional regulator protein & 8.83E-07 & 2.37 & 0.26 & 9.12 & & \\
\hline
\end{tabular}

${ }^{a}$ Average signal intensity $\log _{2}$ values from three experiments.

${ }^{b}$ Transcript ratio between WT and $\triangle h m g A$.

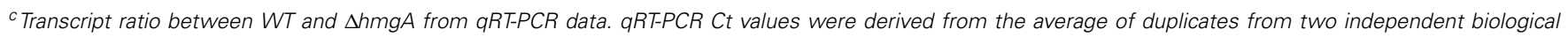
samples.

'Undivisible as no transcript was detected from $\Delta h m g A$.

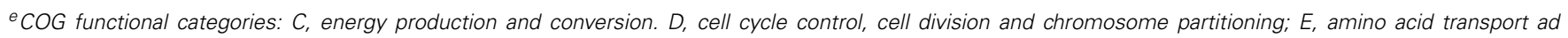

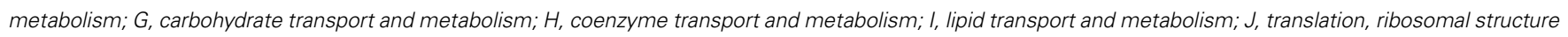

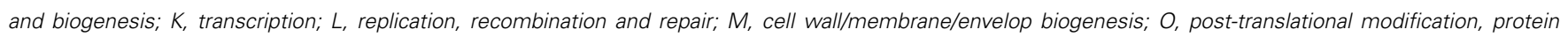

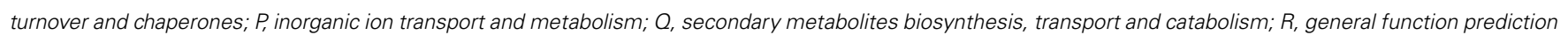
only; $S$, function unknown; $T$, signal transduction mechanisms; $U$, intracellular trafficking, secretion and vesicular transport; $V$, defense mechanisms.

several virulence factors in $\triangle h m g A$. However, with the exception of two [hemolysin (M892_26640) and azurin (M892_25430)], the genes encoding these factors were not differentially expressed suggesting that the decreased virulence potential of $\Delta h m g A$ was not caused by an overt down-regulation of accepted virulence factors.

The expression of bioluminescence has also been associated with virulence in shrimp (Manefield et al., 2000; Phuoc et al., 2009) and indirect evidence has suggested that bioluminescence and a toxic extracellular protein are co-regulated (Manefield et al., 2000). The molecular mechanisms of Vibrio quorum sensing and its resulting bioluminescence have been most extensively studied in $V$. campbellii BAA-1116 and the regulation of luxCD$A B E G H$ gene cluster responsible for bioluminescence is known to be positively regulated by the quorum sensing master regulator LuxR (Bassler et al., 1993; Henke and Bassler, 2004b; Lenz et al., 2004; Waters and Bassler, 2006; Tu and Bassler, 2007). In this study, the bioluminescence output of $\triangle h m g A$ was found to be significantly attenuated and this phenotype could be attributed to the pronounced down-regulation of luxCDABEGH in $\triangle \mathrm{hmgA}$ (Table 2). Furthermore, the gene encoding the high cell density state quorum sensing master regulator LuxR and another quorum sensing regulator (LuxT) (Lin et al., 2000) were also significantly modulated in $\triangle h m g A$. It is estimated that this singular effect on $\operatorname{luxR}$ transcript levels accounted for $40 \%$ of the transcriptional modulation seen in $\triangle h m g A$ as a comparison with the LuxR regulon (manuscript in preparation) revealed 52 modulated genes in common. 
In addition to the lux bioluminescence genes, LuxR has also been shown to regulate the synthesis of storage polyhydroxybutyrates in bioluminescent vibrios (Miyamoto et al., 1998). Polyhydroxybutyrates, the most characterized member of the polyhydroxyalkanoates, are storage polyesters that are produced and accumulate in the bacterial cytosol in carbon-rich environments when other nutrients are limited (Reddy et al., 2003). When available carbon has been exhausted, these storage polymers can be catabolized for carbon and energy. In V. campbellii $\triangle h m g A$, the expression of the polyhydroxybutyrate synthesis gene cluster (phaCDAB) was markedly reduced in comparison to the WT. Thus, a blockage of the tyrosine catabolism pathway and production of pyomelanin not only retarded amino acid degradation but also kept $V$. campbellii from accumulating carbon storage polymers when in a carbon-rich environment. Evidence of these nutrient management deficiencies could be seen in the late stationary phase $\Delta h m g A$ cultures (Figure 2A) where depleted carbon supplies may have contributed to diminished survival.

The transcriptional modulation of members of the LuxR quorum sensing regulon in $\triangle h m g A$, presumably due to reduced luxR transcript levels, was unexpected as HmgA is not known to be a regulatory protein. One possible explanation for this observation may be that extracellular pyomelanin is somehow interfering with the binding of autoinducer molecules to their cognate quorum sensing histidine kinase receptors. Alternatively, membrane-embedded pyomelanin may sufficiently alter membrane structure and impair the binding of autoinducer molecules or subsequent phosphorylation cascade. In either case, the quorum sensing signaling cascade would mimic a low cell density state resulting in the phosphorylation of the response regulator LuxO and activated transcription of regulatory small RNAs (Qrr sRNAs) (Lilley and Bassler, 2000). As the base-pairing of the Qrr sRNAs to $l u x R$ transcripts results in the degradation of $l u x R$ mRNA (Lenz et al., 2004; Tu and Bassler, 2007), pyomelanization may lock the cells in a low cell density state thus, explaining the transcriptome profiling data.

\section{CONCLUSIONS}

In this study, we used the model quorum sensing bacterium $V$. campbellii BAA-1116 to determine how the deletion of the hmgA gene and resulting pyomelanization affected cellular phenotypes, virulence, and transcription. While the material properties of $V$. campbellii pyomelanin were similar to previous descriptions, there did not appear to be a generality of pyomelanin-mediated phenotypes as pyomelanization had either a neutral or deleterious effect on cell survival. Despite the fact that pyomelanin production in other pathogenic bacteria had been shown to increase their virulence and adaptation to chronicity in mammalian infection models, our results using a shrimp infection model indicated that pyomelanized $V$. campbellii were actually less virulent than the isogenic WT strain. These observations may be due in part to the comparatively lesser amount of pyomelanin produced and retained in $V$. campbellii and the effect this production had on cellular metabolism. This was supported by the first transcriptome-level analysis comparing a pyomelaninproducing mutant with its isogenic WT strain. It is also worth noting that the immune responses from different animal models (invertebrate vs. vertebrate) may also play a large role in the differences seen in virulence. $V$. campbellii is primarily an invertebrate animal pathogen and this is the first time that the effect of pyomelanization has been tested in an invertebrate model system (i.e., a natural host organism). The transcriptional profiles demonstrated that the deletion of the $h m g A$ gene led to significantly lower transcript abundance levels of several important metabolic processes that disrupted cellular homeostasis and fitness in stressful environments (e.g., stationary phase). Taken together, these findings may explain why naturally pyomelanized $V$. campbellii or $V$. harveyi have not been identified from the marine environment or infected eukaryotic host organisms.

\section{ACKNOWLEDGMENTS}

This work was funded by the Office of Naval Research via U.S. Naval Research Laboratory core funds. The opinions and assertions contained herein are those of the authors and are not to be construed as those of the U.S. Navy, military service at large, or U.S. Government.

\section{REFERENCES}

Amparyup, P., Charoensapsri, W., and Tassanakajon, A. (2009). Two prophenoloxidases are important for the survival of Vibrio harveyi challenged shrimp Penaeus monodon. Dev. Comp. Immunol. 33, 247-256. doi: 10.1016/j.dci.2008. 09.003

Austin, B., and Zhang, X. H. (2006). Vibrio harveyi: a significant pathogen of marine vertebrates and invertebrates. Lett. Appl. Microbiol. 43, 119-124. doi: 10.1111/j.1472-765X.2006.01989.x

Bassler, B. L. (1999). How bacteria talk to each other: regulation of gene expression by quorum sensing. Curr. Opin. Microbiol. 2, 582-587. doi: 10.1016/S13695274(99)00025-9

Bassler, B. L., Wright, M., Showalter, R. E., and Silverman, M. R. (1993). Intercellular signalling in Vibrio harveyi: sequence and function of genes regulating expression of luminescence. Mol. Microbiol. 9, 773-786. doi: 10.1111/j. 1365-2958.1993.tb01737.x

Cerenius, L., and Soderhall, K. (2004). The prophenoloxidase-activating system in invertebrates. Immunol. Rev. 198, 116-126. doi: 10.1111/j.01052896.2004.00116.x

Charoensapsri, W., Amparyup, P., Hirono, I., Aoki, T., and Tassanakajon, A. (2009). Gene silencing of a prophenoloxidase activating enzyme in the shrimp, Penaeus monodon, increases susceptibility to Vibrio harveyi infection. Dev. Comp. Immunol. 33, 811-820. doi: 10.1016/j.dci.2009.01.006

Coyne, V. E., and Al-Harthi, L. (1992). Induction of melanin biosynthesis in Vibrio cholerae. Appl. Environ. Microbiol. 58, 2861-2865.

Czyz, A., Plata, K., and Wegrzyn, G. (2002). Induction of light emission by luminescent bacteria treated with UV light and chemical mutagens. J. Appl. Genet. 43, 377-389.

Czyz, A., Wrobel, B., and Wegrzyn, G. (2000). Vibrio harveyi bioluminescence plays a role in stimulation of DNA repair. Microbiology 146, 283-288.

Defoirdt, T., Darshanee Ruwandeepika, H. A., Karunasagar, I., Boon, N., and Bossier, P. (2010). Quorum sensing negatively regulates chitinase in Vibrio harveyi. Environ. Microbiol. Rep. 2, 44-49. doi: 10.1111/j.1758-2229.2009.00043.x

Defoirdt, T., Verstraete, W., and Bossier, P. (2008). Luminescence, virulence and quorum sensing signal production by pathogenic Vibrio campbellii and Vibrio harveyi isolates. J. Appl. Microbiol. 104, 1480-1487. doi: 10.1111/j.13652672.2007.03672.x

Henke, J. M., and Bassler, B. L. (2004a). Quorum sensing regulates type III secretion in Vibrio harveyi and Vibrio parahaemolyticus. J. Bacteriol. 186, 3794-3805. doi: 10.1128/JB.186.12.3794-3805.2004

Henke, J. M., and Bassler, B. L. (2004b). Three parallel quorum-sensing systems regulate gene expression in Vibrio harveyi. J. Bacteriol. 186, 6902-6914. doi: 10.1128/JB.186.20.6902-6914.2004

Ji, P. F., Yao, C. L., and Wang, Z. Y. (2011). Reactive oxygen system plays an important role in shrimp Litopenaeus vannamei defense against Vibrio parahaemolyticus and WSSV infection. Dis. Aquat. Organ. 96, 9-20. doi: 10.3354/dao02373 
Katsev, A. M., Wegrzyn, G., and Szpilewska, H. (2004). Effects of hydrogen peroxide on light emission by various strains of marine luminescent bacteria. J. Basic Microbiol. 44, 178-184. doi: 10.1002/jobm.200310330

Kazakov, A. E., Rodionov, D. A., Alm, E., Arkin, A. P., Dubchak, I., and Gelfand, M. S. (2009). Comparative genomics of regulation of fatty acid and branchedchain amino acid utilization in proteobacteria. J. Bacteriol. 191, 52-64. doi: 10.1128/JB.01175-08

Kotob, S. I., Coon, S. L., Quintero, E. J., and Weiner, R. M. (1995). Homogentisic acid is the primary precursor of melanin synthesis in Vibrio cholerae, a Hyphomonas strain, and Shewanella colwelliana. Appl. Environ. Microbiol. 61, 1620-1622.

Kozakiewicz, J., Gajewska, M., Lyzen, R., Czyz, A., and Wegrzyn, G. (2005). Bioluminescence-mediated stimulation of photoreactivation in bacteria. FEMS Microbiol. Lett. 250, 105-110. doi: 10.1016/j.femsle.2005.06.047

Kurowski, B., and Ludwig, B. (1987). The genes of the Paracoccus denitrificans bcl complex. Nucleotide sequence and homologies between bacterial and mitochondrial subunits. J. Biol. Chem. 262, 13805-13811.

Lee, J. H., Park, N. Y., Lee, M. H., and Choi, S. H. (2003). Characterization of the Vibrio vulnificus putAP operon, encoding proline dehydrogenase and proline permease, and its differential expression in response to osmotic stress. J. Bacteriol. 185, 3842-3852. doi: 10.1128/JB.185.13.3842-3852.2003

Lenz, D. H., Mok, K. C., Lilley, B. N., Kulkarni, R. V., Wingreen, N. S., and Bassler, B. L. (2004). The small RNA chaperone Hfq and multiple small RNAs control quorum sensing in Vibrio harveyi and Vibrio cholerae. Cell 118, 69-82. doi: 10.1016/j.cell.2004.06.009

Lilley, B. N., and Bassler, B. L. (2000). Regulation of quorum sensing in Vibrio harveyi by LuxO and sigma-54. Mol. Microbiol. 36, 940-954. doi: 10.1046/j.13652958.2000.01913.x

Lin, B., Wang, Z., Malanoski, A. P., O'Grady, E. A., Wimpee, C. F., Vuddhakul, V., et al. (2010). Comparative genomic analyses identify the Vibrio harveyi genome sequenced strains BAA-1116 and HY01 as Vibrio campbellii. Environ. Microbiol. Rep. 2, 81-89. doi: 10.1111/j.1758-2229.2009.00100.x

Lin, Y. H., Miyamoto, C., and Meighen, E. A. (2000). Cloning and functional studies of a luxO regulator LuxT from Vibrio harveyi. Biochim. Biophys. Acta 1494, 226-235. doi: 10.1016/S0167-4781(00)00236-0

Manefield, M., Harris, L., Rice, S. A., De Nys, R., and Kjelleberg, S. (2000). Inhibition of luminescence and virulence in the black tiger prawn (Penaeus monodon) pathogen Vibrio harveyi by intercellular signal antagonists. Appl. Environ. Microbiol. 66, 2079-2084. doi: 10.1128/AEM.66.5.20792084.2000

Metcalf, W. W., Jiang, W., Daniels, L. L., Kim, S. K., Haldimann, A., and Wanner, B. L. (1996). Conditionally replicative and conjugative plasmids carrying lac $Z$ alpha for cloning, mutagenesis, and allele replacement in bacteria. Plasmid 35, 1-13. doi: 10.1006/plas.1996.0001

Miyamoto, C. M., Sun, W., and Meighen, E. A. (1998). The LuxR regulator protein controls synthesis of polyhydroxybutyrate in Vibrio harveyi. Biochim. Biophys. Acta 1384, 356-364. doi: 10.1016/S0167-4838(98)00028-4

Mok, K. C., Wingreen, N. S., and Bassler, B. L. (2003). Vibrio harveyi quorum sensing: a coincidence detector for two autoinducers controls gene expression. EMBO J. 22, 870-881. doi: 10.1093/emboj/cdg085

Nakada, Y., Nishijyo, T., and Itoh, Y. (2002). Divergent structure and regulatory mechanism of proline catabolic systems: characterization of the putAP proline catabolic operon of Pseudomonas aeruginosa PAO1 and its regulation by PruR, an AraC/XylS family protein. J. Bacteriol. 184, 5633-5640. doi: 10.1128/JB.184.20.5633-5640.2002

Natrah, F. M., Ruwandeepika, H. A., Pawar, S., Karunasagar, I., Sorgeloos, P., Bossier, P., et al. (2011). Regulation of virulence factors by quorum sensing in Vibrio harveyi. Vet. Microbiol. 154, 124-129. doi: 10.1016/j.vetmic.2011.06.024

Phuoc, L. H., Defoirdt, T., Sorgeloos, P., and Bossier, P. (2009). Virulence of luminescent and non-luminescent isogenic vibrios toward gnotobiotic Artemia franciscana larvae and specific pathogen-free Litopenaeus vannamei shrimp. J. Appl. Microbiol. 106, 1388-1396. doi: 10.1111/j.1365-2672.2008.04107.x

Reddy, C. S., Ghai, R., Rashmi, and Kalia, V. C. (2003). Polyhydroxyalkanoates: an overview. Bioresour. Technol. 87, 137-146. doi: 10.1016/S0960-8524(02)00212-2

Reed, L. J., and Muench, H. (1938). A simple method of estimating fifty percent end point. Am. J. Hyg. 27, 493-497.

Rodriguez-Rojas, A., Mena, A., Martin, S., Borrell, N., Oliver, A., and Blazquez, J. (2009). Inactivation of the $h m g A$ gene of Pseudomonas aeruginosa leads to pyomelanin hyperproduction, stress resistance and increased persistence in chronic lung infection. Microbiology 155, 1050-1057. doi: 10.1099/mic.0.024745-0

Rubin, R. A. (2009). A first principles approach to differential expression in microarray data analysis. BMC Bioinform. 10:292. doi: 10.1186/1471-210510-292

Schmaler-Ripcke, J., Sugareva, V., Gebhardt, P., Winkler, R., Kniemeyer, O., Heinekamp, T., et al. (2009). Production of pyomelanin, a second type of melanin, via the tyrosine degradation pathway in Aspergillus fumigatus. Appl. Environ. Microbiol. 75, 493-503. doi: 10.1128/AEM.02077-08

Simon, J. (2002). Enzymology and bioenergetics of respiratory nitrite ammonification. FEMS Microbiol. Rev. 26, 285-309. doi: 10.1111/j.1574-6976.2002. tb00616.x

Szpilewska, H., Czyz, A., and Wegrzyn, G. (2003). Experimental evidence for the physiological role of bacterial luciferase in the protection of cells against oxidative stress. Curr. Microbiol. 47, 379-382. doi: 10.1007/s00284-002-4024-y

Thompson, F. L., Iida, T., and Swings, J. (2004). Biodiversity of vibrios. Microbiol. Mol. Biol. Rev. 68, 403-431. doi: 10.1128/MMBR.68.3.403-431.2004

Tu, K. C., and Bassler, B. L. (2007). Multiple small RNAs act additively to integrate sensory information and control quorum sensing in Vibrio harveyi. Genes Dev. 21, 221-233. doi: 10.1101/gad.1502407

Turick, C. E., Beliaev, A. S., Zakrajsek, B. A., Reardon, C. L., Lowy, D. A., Poppy, T. E., et al. (2009). The role of 4-hydroxyphenylpyruvate dioxygenase in enhancement of solid-phase electron transfer by Shewanella oneidensis MR-1. FEMS Microbiol. Ecol. 68, 223-225. doi: 10.1111/j.1574-6941.2009.00670.x

Turick, C. E., Tisa, L. S., and Caccavo, F., Jr. (2002). Melanin production and use as a soluble electron shuttle for Fe(III) oxide reduction and as a terminal electron acceptor by Shewanella algae BrY. Appl. Environ. Microbiol. 68, 2436-2444. doi: 10.1128/AEM.68.5.2436-2444.2002

Valeru, S. P., Rompikuntal, P. K., Ishikawa, T., Vaitkevicius, K., Sjoling, A., Dolganov, N., et al. (2009). Role of melanin pigment in expression of Vibrio cholerae virulence factors. Infect. Immun. 77, 935-942. doi: 10.1128/IAI. 00929-08

Wang, R., Wang, H., Zhou, H., Wang, Y., Yue, J., Diao, B., et al. (2011). Characters of homogentisate oxygenase gene mutation and high clonality of the natural pigment-producing Vibrio cholerae strains. BMC Microbiol. 11:109. doi: 10.1186/1471-2180-11-109

Warrens, A. N., Jones, M. D., and Lechler, R. I. (1997). Splicing by overlap extension by PCR using asymmetric amplification: an improved technique for the generation of hybrid proteins of immunological interest. Gene 186, 29-35. doi: 10.1016/S0378-1119(96)00674-9

Waters, C. M., and Bassler, B. L. (2006). The Vibrio harveyi quorum-sensing system uses shared regulatory components to discriminate between multiple autoinducers. Genes Dev 20, 2754-2767. doi: 10.1101/gad.1466506

Youngchim, S., Morris-Jones, R., Hay, R. J., and Hamilton, A. J. (2004). Production of melanin by Aspergillus fumigatus. J. Med. Microbiol. 53, 175-181. doi: 10.1099/jmm.0.05421-0

Zughaier, S. M., Ryley, H. C., and Jackson, S. K. (1999). A melanin pigment purified from an epidemic strain of Burkholderia cepacia attenuates monocyte respiratory burst activity by scavenging superoxide anion. Infect. Immun. 67, 908-913.

Conflict of Interest Statement: The authors declare that the research was conducted in the absence of any commercial or financial relationships that could be construed as a potential conflict of interest.

Received: 18 September 2013; paper pending published: 21 October 2013; accepted: 22 November 2013; published online: 11 December 2013.

Citation: Wang Z, Lin B, Mostaghim A, Rubin RA, Glaser ER, Mittraparp-arthorn P, Thompson JR, Vuddhakul V and Vora GJ (2013) Vibrio campbellii hmgA-mediated pyomelanization impairs quorum sensing, virulence, and cellular fitness. Front. Microbiol. 4:379. doi: 10.3389/fmicb.2013.00379

This article was submitted to Aquatic Microbiology, a section of the journal Frontiers in Microbiology.

Copyright (c) 2013 Wang, Lin, Mostaghim, Rubin, Glaser, Mittraparp-arthorn, Thompson, Vuddhakul and Vora. This is an open-access article distributed under the terms of the Creative Commons Attribution License (CC BY). The use, distribution or reproduction in other forums is permitted, provided the original author(s) or licensor are credited and that the original publication in this journal is cited, in accordance with accepted academic practice. No use, distribution or reproduction is permitted which does not comply with these terms. 